\title{
RED DE CENTROS EDUCATIVOS Y DESEQUILIBRIOS TERRITORIALES. EL CASO DE ARAGÓN*
}

\author{
Severino Escolano Utrilla, Enrique Ruiz Budría y Eugenio Climent López \\ Departamento de Geografía y Ordenación del Territorio (Universidad de Zaragoza) \\ Calle Pedro Cerbuna, no 12; 50008-Zaragoza \\ severino@unizar.es eruiz@unizar.es edliment@unizar.es
}

\begin{abstract}
Resumen: En este trabajo se analiza la organización territorial de los servicios educativos en Aragón. Esta Comunidad se caracteriza por fuertes desequilibrios territoriales, como pone de relieve la existencia de extensas áreas de muy baja densidad de población, dotadas de redes de asentamientos muy laxas y de infraestructuras de transporte poco densas y de baja capacidad, lo cual dificulta la prestación, en condiciones de equidad y eficiencia territorial, de los servicios educativos a los que tienen derecho todos los ciudadanos. En este contexto se estudia la adecuación de la red de centros escolares a la estructura territorial. Los centros privados se localizan siguiendo criterios de demanda y por ello se ubican casi exclusivamente en los núcleos urbanos, mientras que la red de centros públicos alcanza a la mayor parte del territorio.
\end{abstract}

Palabras clave: desequilibrios territoriales, red de centros escolares, Aragón.

\begin{abstract}
In this paper, territorial organisation of educational services in Aragon is studied. This Spanish region is characterized by strong territorial imbalances, as indicated by areas with a very low population density, a feeble settlement network and a transport infrastructure of low density and capability. These imbalances make difficult to provide the educational services that citizens have the right to access in conditions of territorial equity and efficiency. In this context, the suitability of school network to territorial structure is analysed. The great majority of the private centres, according to demand criteria, are located in the urban settlements. Otherwise, the network of public centres reaches the most part of the territory.
\end{abstract}

Key words: territorial imbalances, school network, Aragon.

* Recibido: 21-03-03. Aceptado: 07-07-05. 


\section{Introducción}

No parece necesario insistir en el valor estratégico de la educación y la formación en las sociedades modernas, caracterizadas por la elevada complejidad de las relaciones sociales y de la tecnología, como tampoco en la universalidad del derecho a la educación y los principios de calidad, equidad y eficiencia que deben inspirar la planificación y prestación de los servicios educativos y formativos.

Sin embargo, ese derecho no será universal de modo efectivo si no se dan las condiciones prácticas que permitan a toda la población el acceso a dichos servicios. Por ello un aspecto fundamental de la política educativa en los países democráticos es la organización de una red de centros capaz de proporcionar a todos sus ciudadanos una educación y formación acorde con los principios citados, sin discriminación alguna.

El diseño de una red de dichas características debe tener en cuenta múltiples variables, entre ellas las territoriales, que son las que constituyen el ámbito más específico de la investigación geográfica. Los territorios son diversos y asegurar a todos sus habitantes, sin discriminación, el acceso a la educación exige tener en cuenta la diversidad territorial, lo cual plantea problemas complejos. El espacio uniforme, la llanura isótropa, es una situación ideal y, por ello, adecuada para la elaboración de modelos teóricos, pero el planificador se enfrenta a situaciones de gran heterogeneidad para las que los modelos son una referencia, no una receta.

El objetivo de este artículo es describir y valorar cómo se resuelve o se intenta resolver, mediante la red de centros educativos, el problema de garantizar el derecho a la educación de todos los habitantes de un territorio caracterizado por fuertes desequilibrios territoriales (muy alejado, por tanto, de la llanura isótropa), sin que se vean discriminados por su lugar de residencia. Para ello se toma como caso de estudio la comunidad autónoma de Aragón.

\section{Servicios educativos y territorio}

Uno de los ámbitos centrales de investigación de la geografía es el organizado en torno al análisis y estudio de las relaciones espaciales de los diferentes elementos y objetos que constituyen un fenómeno o problema social. En el caso del derecho a la educación, la proximidad o lejanía de los centros de estudio respecto al domicilio de los estudiantes y las dificultades asociadas a los desplazamientos, modifican en la práctica las condiciones de igualdad de oportunidades hasta producir en algunos casos desequilibrios inadmisibles. 
La geografía ha elaborado teorías, conceptos, métodos y técnicas para describir y modelar la organización territorial de las redes de servicios y para dar cuenta de sus carencias y grado de eficacia. Hay un sólido corpus teórico, elaborado por la geografía teorético-cuantitativa de la tradición neo-positivista, en cuyos orígenes está la teoría de los lugares centrales, formulada por Christaller (1966), donde se establecen y sistematizan los principios que explican la organización interurbana (e intra-urbana) del comercio y los servicios. Ella ha sido el punto de partida de desarrollos posteriores, entre cuyos jalones más significativos se encuentran los trabajos de Berry (1972) sobre los centros de mercado y los de Beavon (1981) sobre las actividades terciarias.

De dicho corpus teórico se desprende que la distribución espacial de los asentamientos es el primer condicionante de la localización de los servicios, en general, y de los centros educativos, en particular. Los asentamientos se estructuran en redes organizadas jerárquicamente en función de sus habitantes y la cantidad y variedad de servicios que ofrecen. Dicha jerarquía abarca desde las grandes aglomeraciones urbanas de millones de personas hasta las pequeñas aldeas donde conviven unas pocas familias. Dado su elevado número de habitantes, o sea, de potenciales usuarios, las primeras disponen de toda clase de servicios, incluidos los grandes centros de enseñanza superior, mientras que las segundas concentran un volumen de demanda tan escaso que difícilmente en ellos se pueden mantener servicios o equipamientos, por lo que la única alternativa viable en numerosas ocasiones es ubicar éstos en unos núcleos de población seleccionados y que los habitantes de los demás se desplacen a ellos, procurando minimizar los costes, económicos o de otro tipo, de desplazamiento del conjunto de los usuarios (Moreno, 1995; Pitarch, 2000).

Por eso, para garantizar a los ciudadanos la prestación de los servicios educativos es fundamental contar con una red de infraestructuras de transportes y comunicaciones que asegure la movilidad de las personas y de la información. La jerarquía y características de las redes de transporte condicionan la forma y tamaño de las áreas de servicio y privilegian determinados ejes y centros en detrimento de otros. Las modernas redes de comunicación, por su parte, facilitan el acceso a la información y posibilitan el uso de una creciente variedad de recursos educativos y la aplicación de nuevos métodos de aprendizaje.

El instrumento básico para la planificación territorial de los sistemas educativos lo constituye el "mapa escolar", como propugna el trabajo clásico de Hallak (1978). Este documento ha evolucionado con el tiempo: las primeras versiones se limitan por lo general a recoger el volumen y localización de los recursos educativos en textos, tablas, gráficos y cartografía sencilla (los mapas de estos trabajos están directamente inspirados en los que representan las áreas de atracción comercial, que consisten en el trazado de líneas que unen un centro de comercio y servicios con los núcleos de donde acuden compradores y usuarios); los modelos recientes añaden a lo anterior datos relativos a las circunstancias de la demanda y su evolución previsible, al trans- 
porte y a otras elaboraciones analíticas. El volumen de información manejada, asî como su diversidad de tratamientos (analíticos, gráficos, cartográficos), hacen necesario recurrir a programas informáticos para su gestión. La representación cartográfica es crucial en estos informes, lo que justifica que forme parte de su denominación genérica: "mapa escolar".

La conveniencia de reordenar los sistemas educativos para ajustarlos a la situación socioeconómica y político-administrativa surgida por el traspaso de las competencias de educación a las Comunidades Autónomas, ha promovido en las dos últimas décadas la elaboración de varios "mapas escolares", entre los que pueden citarse los de Cataluña y la provincia de Barcelona (de 1989 y 1990, respectivamente), los de Valencia (Salom y Albertos, 2000) y Cantabria (Reques, 2000). Para el caso de Aragón se cuenta con algunos trabajos parciales, dedicados a niveles educativos o a áreas concretas y con dos trabajos generales separados por algo más de una década: Garcés (1991) y Escolano et al. (2003).

Los servicios educativos en España son gestionados por algunas instituciones privadas y por las comunidades autónomas, si bien la ordenación de la estructura general del sistema educativo es competencia del estado. Dicha estructura, tal como la establece la Ley Orgánica 10/2002 de Calidad de la Educación, está compuesta por los siguientes niveles: la educación superior, encomendada a las universidades, que cuentan con una amplia autonomía; la educación preescolar, que tiene como finalidad la atención educativa y asistencial a la primera infancia; las enseñanzas de régimen especial, que cubren campos específicos del saber, como la música, las artes y los idiomas, y, finalmente, las enseñanzas escolares de régimen general.

Estas últimas comprenden unos niveles educativos obligatorios y otros voluntarios. Los primeros son la Educación Primaria y la Educación Secundaria Obligatoria (ESO), que se cursan con carácter general entre los seis y los doce años y entre los doce y los dieciséis, respectivamente. Los niveles voluntarios se ubican antes y después de los obligatorios: antes de la educación primaria se sitúa la Educación Infantil, que acoge a los niños de tres a seis años; después de la ESO el alumno puede optar por el Bachillerato (dos años) o la Formación Profesional (FP) de grado medio (un año o año y medio). Los alumnos que terminan el Bachillerato pueden acceder a la FP de grado superior o a la universidad.

Este trabajo se circunscribe a las enseñanzas escolares de régimen general, pues es en ellas, fundamentalmente en los niveles obligatorios, donde se manifiesta de forma más acuciante el problema de organizar en el territorio una red de centros capaz de prestar a toda la población un servicio de calidad de manera equitativa y eficiente.

En el apartado 3 se analiza la localización espacial de los sujetos del derecho a la educación, que son los usuarios potenciales de los servicios educativos, lo cual equivale a considerar la localización de la población, pues todos los ciudadanos tienen 
ese derecho, pero especialmente la de los niños y jôvenes que están obligados por ley a recibir dichos servicios. En el apartado 4 se analiza la red de centros escolares en relación con la red de asentamientos, calibrando el grado de adecuación de ambas, y en el 5 se analiza la manera en que la red de carreteras y los servicios de transporte escolar tratan de acercar los centros a los usuarios. En el apartado 5 se realizan algunas comparaciones con otros servicios a la población dentro de la propia comunidad autónoma de Aragón y con la red escolar de otra comunidad autónoma, terminando con un apartado de conclusiones.

\section{La población escolar y la red de asentamientos de población en Aragón}

La distribución de la población aragonesa en el espacio nunca ha sido homogénea, dada la localización irregular de los recursos, pero la desigual incidencia espacial de la emigración en el pasado siglo, con sus secuelas de declive y envejecimiento, ha acentuado los contrastes territoriales. El problema fundamental, como evidencia el mapa de la figura 1, es la existencia de extensas áreas con densidad de población inferior a 15 e incluso 5 habitantes por kilómetro cuadrado, lo cual implica serias dificultades para el mantenimiento de las redes de servicios.

La red de asentamientos conserva, en sus trazos generales, la configuración espacial tradicional, adaptada a los rasgos estructurales de las grandes unidades de relieve: en los Pirineos y en el Sistema Ibérico, al norte y sur de la región, respectivamente, el relieve quebrado, con alternancia de valles y sierras, orienta la ocupación lineal del territorio, siguiendo los cauces fluviales, a la vez que empuja a la dispersión de la población en pequeños asentamientos distribuidos por valles y laderas. Por el contrario, en la Depresión del Ebro, que ocupa la franja central de la región, el relieve llano permite la circulación en todas direcciones, favoreciendo la concentración de la población en asentamientos mayores y la disposición más compacta de éstos sobre el terreno. Esta configuración tradicional se vio fuertemente alterada a lo largo del siglo XX por la emigración, produciéndose el abandono de bastantes núcleos de población y la reducción drástica del censo de otros muchos.

El mapa de la figura 1 muestra con claridad el contraste entre las áreas montañosas, con un elevado número de asentamientos de pequeño tamaño dispersos por el territorio, y el área llana, donde los asentamientos son de mayor tamaño y su número sensiblemente menor. En las áreas de montaña la reducida dimensión poblacional de la mayoría de los asentamientos es el factor que se opone con mayor fuerza a la dispersión territorial de los servicios, pues es difícil encontrar agrupaciones de núcleos que, estando separados por una distancia razonable, contengan un volumen de población suficiente para el mantenimiento auto-sostenido de los mismos. 


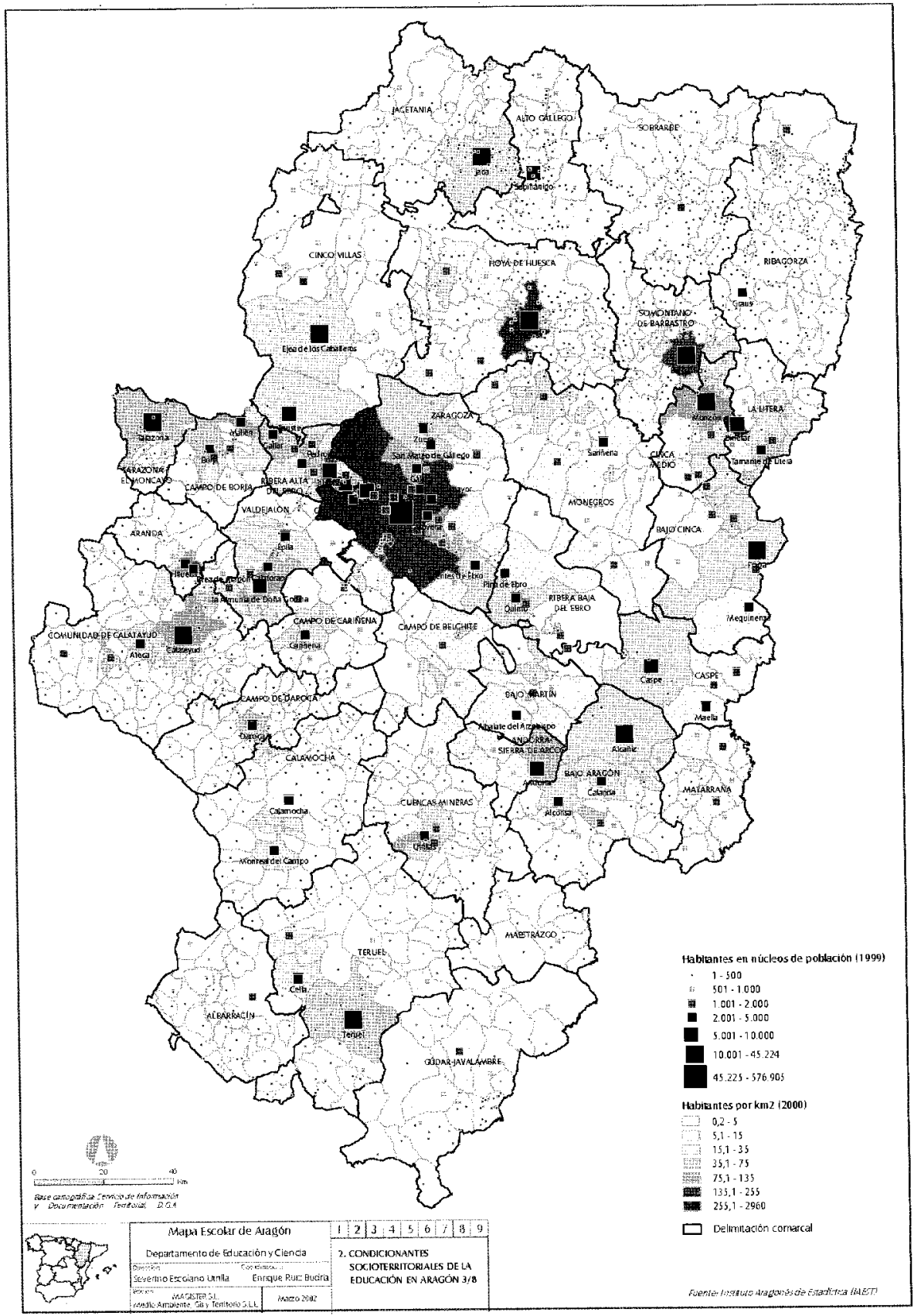

Figura 1. Densidad de población por municipios y núcleos de población por tamaño. Fuente: Escolano et al. (2003, p. 49). 
Tabla 1. Núcleos de población según el número de habitantes (1999).

\begin{tabular}{|lcrrrrr|}
\hline $\begin{array}{l}\text { Habitantes } \\
\text { (intervalos) }\end{array}$ & $\begin{array}{c}\text { Número } \\
\text { de núcleos }^{*}\end{array}$ & $\begin{array}{c}\text { Número } \\
\text { de Habitantes }\end{array}$ & $\begin{array}{c}\text { Alumnos } \\
\text { de EO* }\end{array}$ & $\begin{array}{c}\text { \% sobre } \\
\text { núcleos }\end{array}$ & $\begin{array}{c}\text { \% sobre } \\
\text { población }\end{array}$ & $\begin{array}{r}\% \text { sobre } \\
\text { alumnos }\end{array}$ \\
\hline $1-100$ & 675 & 25.379 & 106 & 51,3 & 2,2 & 0,1 \\
$101-250$ & 282 & 43.430 & 984 & 21,4 & 3,7 & 0,9 \\
$251-500$ & 157 & 55.870 & 1.922 & 11,9 & 4,8 & 1,7 \\
$501-1.000$ & 95 & 68.369 & 2.986 & 7,2 & 5,9 & 2,7 \\
$1.001-2.000$ & 58 & 78.907 & 6.552 & 4,4 & 6,8 & 5,9 \\
$2.001-5.000$ & 29 & 82.777 & 11.244 & 2,2 & 7,1 & 10,2 \\
$5.001-10.000$ & 9 & 63.605 & 7.664 & 0,7 & 5,4 & 6,9 \\
$10.001-50.000$ & 10 & 174.601 & 22.179 & 0,8 & 15,0 & 20,1 \\
Zaragoza & 1 & 574.819 & 56.864 & 0,1 & 49,2 & 51,5 \\
Total Aragón & 1.316 & 1.167 .757 & 110.501 & 100,0 & 100,0 & 100,0 \\
\hline
\end{tabular}

* No se dispone de información demográfica relativa a 29 núcleos

** Alumnos de enseñanza obligatoria residentes en los núcleos de población (los datos corresponden al año 2002)

Fuentes: Instituto Aragonés de Estadística (IAEST) y Departamento de Educación y Ciencia del Gobierno de Aragón.

En la tabla 1 queda patente el fuerte desequilibrio de la red de asentamientos: por una parte, el mayor de ellos, Zaragoza, concentra el 49,2 por ciento de la población regional $\mathrm{y}$, por otra parte, los 675 con menos de 100 habitantes, que constituyen el 51,3 por ciento de los asentamientos, albergan sólo al 2,2 por ciento de la población.

En los asentamientos rurales, que son el 96 por ciento del total, vive menos de la cuarta parte de la población aragonesa. Los de mayor tamaño, es decir, los que tienen una población comprendida entre mil y dos mil habitantes, son los más adecuados para albergar centros de servicios básicos, pero en Aragón hay sólo 58, el 4 por ciento del total, que albergan al 7 por ciento de los habitantes de la región. En la figura 1 puede comprobarse que hay grandes extensiones, sobre todo en las áreas de montaña del norte y del sur, que carecen de núcleos de población de este tamaño.

Los núcleos intermedios, cuyo cometido en la vertebración del territorio es esencial, son todavía más escasos y entre ellos los que tienen de cinco a diez mil habitantes, que por su tamaño y funciones se aproximan a los núcleos urbanos, forman un grupo muy reducido.

La red urbana comprende los niveles superiores de la jerarquía de asentamientos, los que tienen más de diez mil habitantes. En el caso de Aragón se presenta como fuertemente primacial, pues la ciudad principal, Zaragoza, supera holgadamente el medio millón de habitantes, pero la segunda, Huesca, no llega a los cincuenta mil. Esta última y Teruel, dotadas de los servicios administrativos y de otros tipos característicos de las capitales de provincia, forman el segundo nivel jerárquico, por debajo 
del cual aparece un grupo de 8 pequeñas ciudades, cuya población varía entre diez mil y veinte mil habitantes. En la figura 1 puede comprobarse la escasa densidad de la red urbana aragonesa, así como la ausencia casi total de este tipo de núcleos en las áreas montañosas.

La red de asentamientos, en general, pero de manera muy especial la red urbana, constituye el armazón básico de la ordenación del territorio, por lo que una estructura desequilibrada, como la de Aragón, es un excelente indicador de la existencia de desequilibrios territoriales.

El Gobiemo regional inició hace unos años un ambicioso proceso de ordenación territorial en el que se estableció la comarca como la unidad espacial básica para, entre otras cosas, la concepción y ejecución coordinada de los planes de servicios e infraestructuras. Los servicios definen lo que Jean (1995) denomina "cuencas de vida", concepto sintético que recoge la relación dialéctica que se establece entre las comunidades y sus territorios en la escala espacial en que transcurre el ejercicio cotidiano de vivir. Las comarcas establecidas en Aragón podrían aproximarse a este concepto.

El problema al que se enfrenta esta política en Aragón es que sólo en 11 de las 33 comarcas establecidas hay una ciudad, a las que cabe añadir otras 6 que cuentan con un núcleo de entre cinco mil y diez mil habitantes. En otras 10 comarcas los asentamientos de mayor tamaño no alcanzan el umbral de los cinco mil habitantes y en las 6 restantes ni siquiera el de los dos mil, lo cual significa que todos sus núcleos de población corresponden a la categoría de rurales.

Trazada así la panorámica de la distribución espacial de la población aragonesa, hay que señalar que entre la población total y la población escolar hay diferencias significativas, como se aprecia en la tabla 1 , en la que figura también el número de alumnos de educación obligatoria que reside en los núcleos de población ${ }^{1}$. Los 675 asentamientos con menos de cien habitantes tienen una población escolar en los niveles obligatorios de sólo 106 alumnos en total, lo cual significa que en la gran mayoría de los mismos no hay ningún residente de edad comprendida entre seis y dieciséis años. Por el contrario, en la ciudad de Zaragoza se concentran 56.864 alumnos, que equivalen al 51,2 por ciento del total regional.

Estas cifras pueden ponerse en una perspectiva temporal de casi tres décadas gracias a Garcés (1991: 574). Comparando la información aportada por éste con la de la tabla 1 se comprueba que a lo largo del tiempo ha habido una clara tendencia a variar la proporción de escolares residentes en los núcleos de población según el tamaño, de manera que cuanto menor es éste más se ha reducido la proporción ${ }^{2}$ : así, por ejemplo, los escolares residentes en núcleos de menos de 100 habitantes han pasado de ser el 1 por ciento del total en 1975 a sólo el 0,1 por ciento en 2002; los residentes en núcleos de 500 a mil habitantes han pasado en las mismas fechas del 6,7 al 2,7 
por ciento. De manera consecuente con lo anterior, cuanto mayor es el tamaño de los núcleos de población más se ha incrementado la proporción de escolares residentes: en los de 5.000 a 10.000 habitantes ha pasado del 5,3 por ciento en 1975 al 6,9 en 2002; por lo que respecta a los núcleos urbanos (el citado trabajo presenta la información de este grupo de asentamientos de forma agregada) ha pasado en esas mismas fechas del 65,9 al 71,6 por ciento.

Una comparación entre los porcentajes de la población total y la población escolar aportados por la tabla 1 revela que aquéllos son superiores a éstos en los núcleos rurales y al revés en los intermedios y urbanos. Estos datos, plenamente coherentes con la tendencia apuntada en el párrafo anterior, reflejan con claridad el mayor grado de envejecimiento de la población rural, pues cuanto menor es el tamaño de los asentamientos menor es el porcentaje de población escolar. La población aragonesa tiene un grado de envejecimiento superior a la media nacional ${ }^{3}$ y su crecimiento a lo largo de las dos últimas décadas ha sido muy próximo al estancamiento, por lo que ese sobre-envejecimiento de la población rural dibuja un panorama muy negativo respecto a la evolución futura de la población.

En definitiva, en Aragón hay extensas áreas de muy baja densidad de población y estructuras demográficas fuertemente envejecidas, cuyos habitantes viven dispersos en asentamientos de pequeño tamaño. En ellas los centros educativos alcanzan umbrales de demanda adecuados sólo cuando sus áreas de servicio tienen superficies muy grandes, con el consiguiente incremento de las distancias a recorrer por los usuarios potenciales.

\section{La red de centros escolares en Aragón}

Una vez analizada la distribución espacial de los asentamientos en que habitan los usuarios de los servicios educativos, procede analizar la de los centros que prestan dichos servicios; en otras palabras y dado que este trabajo se circunscribe a las enseñanzas escolares de régimen general, la distribución espacial de los centros escolares.

El ordenamiento legal vigente en España establece que la educación es un servicio público que puede ser prestado tanto por las instituciones del estado, que en el caso de España son las administraciones regionales de las comunidades autónomas, como por instituciones privadas. Esto hace que, atendiendo a su titularidad, existan dos redes de centros escolares, la pública y la privada. Partiendo, a modo de hipótesis, de que los criterios de localización de centros escolares pueden ser distintos en ambas clases de titulares, se realizará un análisis diferenciado de ambas redes. 


\subsection{La red privada}

Puede observarse en la figura 2, donde se representan los centros escolares de régimen general clasificados por titularidad ${ }^{4}$ y tamaño de los asentamientos, que la iniciativa privada está prácticamente ausente de los de menor número de habitantes: no gestiona ningún centro en los núcleos de población de hasta 100 habitantes y sólo uno en cada grupo de 101-250, 251-500 y 501-1.000, que sumados a los ocho del grupo de 1.001 a 2.000 hacen un total de 11 centros educativos privados en el medio rural, frente a 158 de titularidad pública. Dos de esos centros privados se localizan en asentamientos del área peri-urbana de Zaragoza y los nueve restantes en zonas problemáticas por la debilidad de sus redes de asentamientos ${ }^{5}$.

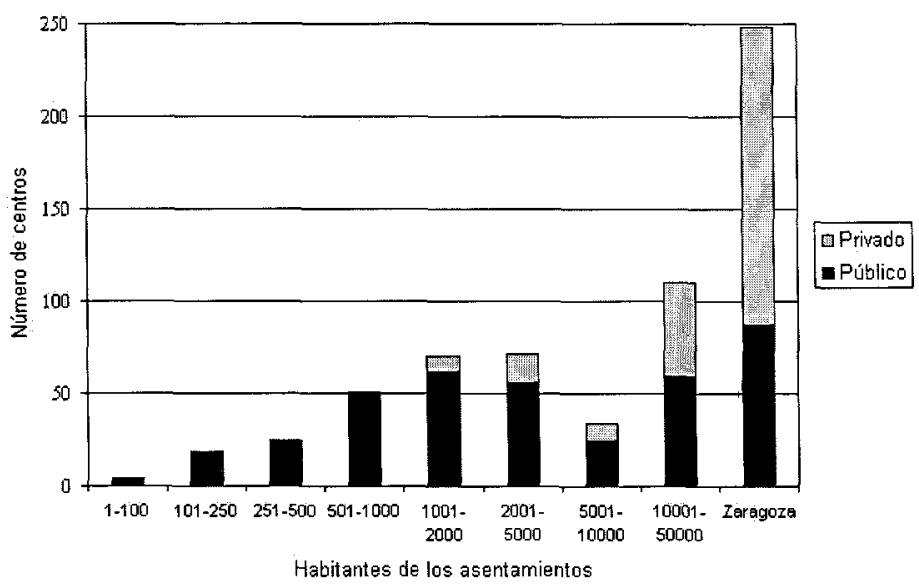

Figura 2. Centros de enseñanza de régimen general según titular y tamaño de asentamientos (2002). Fuente: Gobierno de Aragón. Departamento de Educación y Ciencia.

En los núcleos de población intermedios se incrementa la presencia de centros privados, que suman un total de 24 , pero los de titularidad pública son 81 , por lo que también es patente el predominio de ésta en dichos asentamientos, aunque las diferencias no son tan marcadas como en el grupo anterior. En los asentamientos urbanos de diez mil a cincuenta mil habitantes las cifras casi se igualan: 59 centros públicos, frente a 51 privados. Finalmente, en Zaragoza hay un claro predominio de los centros privados respecto a los públicos: 161 por 87 .

De todo ello se desprende que la red privada es casi exclusivamente urbana y además está muy fuertemente polarizada en Zaragoza, donde se localiza el 65 por ciento 
de los centros privados que ofrecen enseñanzas de régimen general. Si a esta cifra se añade el 20 por ciento que corresponde a las demás ciudades se obtiene el 85 por ciento de los centros de dicha red, lo cual hace que su presencia en los núcleos intermedios y rurales sea casi testimonial.

Si se comparan los porcentajes de centros ubicados en Zaragoza y demás asentamientos urbanos con los de la población que reside en ellos (tabla 1) se ve que la iniciativa privada, en el caso de Aragón, distribuye sus centros educativos de forma desequilibrada en favor de los núcleos de mayor tamaño, guiándose por criterios de demanda y no de equidad territorial. Contrastando esta situación con la que presenta Garcés (1991: 572) para los años finales de la década de los ochenta, se observa que no ha habido variaciones significativas.

\subsection{La red pública}

En contraste con ello, la administración pública concentra en Zaragoza un porcentaje de sus centros educativos muy inferior al de la población que reside en la capital, mientras que en los núcleos de quinientos a cinco mil habitantes el porcentaje de centros es sensiblemente mayor que el de la población residente; en los demás grupos de asentamientos ambos porcentajes se aproximan mucho. De ello se desprende que la administración pública aplica criterios de equidad territorial al decidir la localización de sus centros escolares

Los centros públicos se dividen en dos grandes grupos, según los niveles educativos que en ellos se imparten: por un lado están los de educación infantil y primaria y, por otro, los de educación secundaria, donde se imparten ESO, Bachillerato y FP.

Aunque la infantil no es un nivel educativo obligatorio, su oferta está muy estrechamente asociada a la de primaria, impartiéndose normalmente en los mismos centros. De hecho, como puede verse en la tabla 2, dentro de la red pública de Aragón, sólo hay 4 Escuelas de Educación Infantil, que se dedican exclusivamente a dicho nivel, frente a 274 colegios que se dedican conjuntamente a los dos niveles. Dentro de éstos cabe distinguir los Colegios de Educación Infantil y Primaria y los Centros Rurales Agrupados (CRA).

Los colegios tienen todas sus instalaciones (aulas, laboratorios, biblioteca, gimnasio, comedor, oficinas y demás) concentradas en el mismo punto, respondiendo por lo general a la imagen clásica de colegio: uno o más edificios y un patio de recreo. Se localizan en asentamientos de todos los tamaños, como puede verse en la tabla 2 $\mathrm{y}$ en la figura 3. 


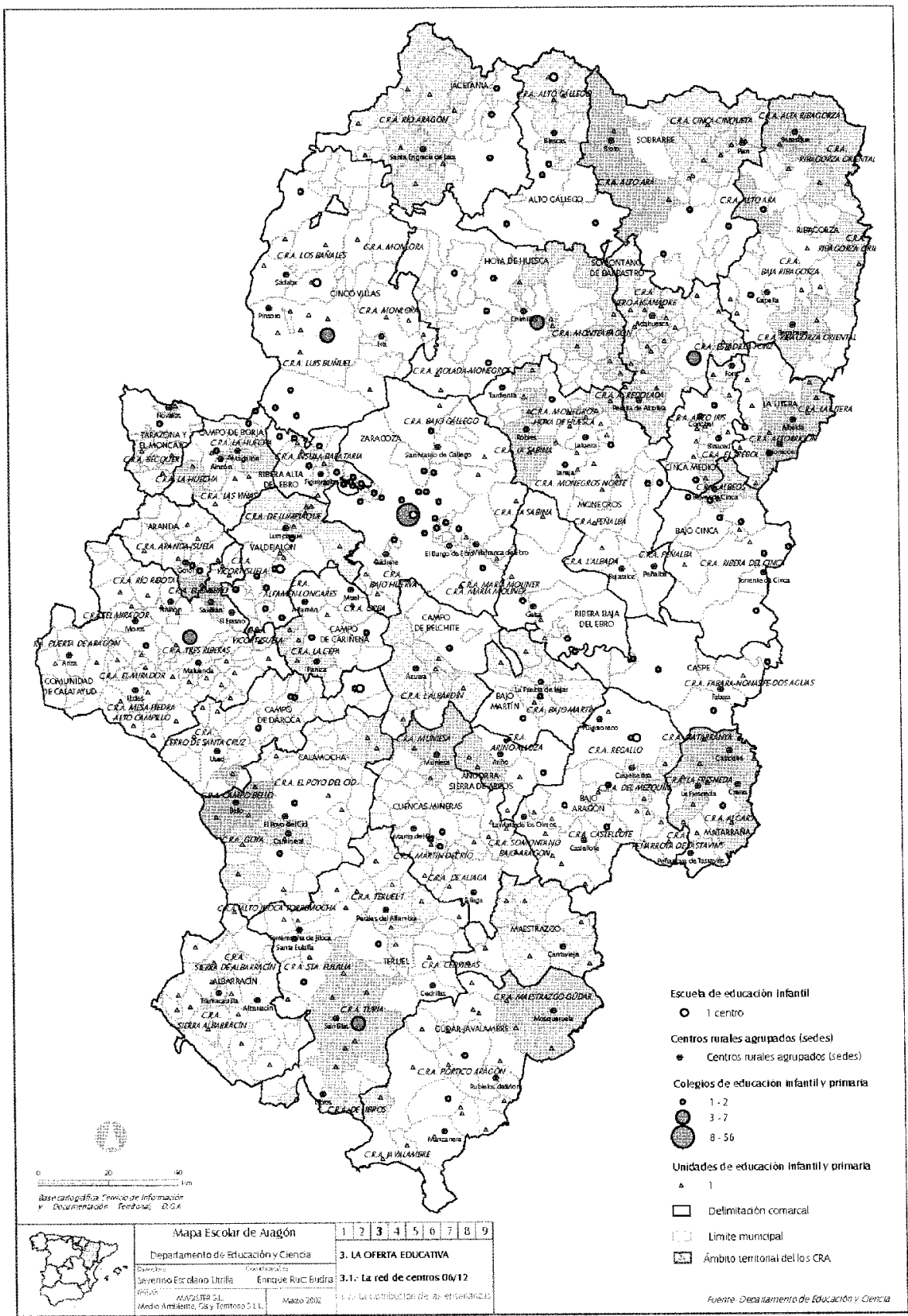

Figura 3. Centros públicos de educación infantil y primaria. Fuente: Escolano et al. (2003, p. 65). 
Tabla 2. Centros de titularidad pública según el tamaño de los núcleos de población (2002).

\begin{tabular}{|l|c|c|c|c|c|c|c|c|c|c|}
\hline \multirow{2}{*}{ Tipos de centro } & \multicolumn{6}{|c|}{ Habitantes de los núcleos de población } \\
\cline { 2 - 11 } & $\mathbf{1}$ & $\mathbf{1 0 1}$ & $\mathbf{2 5 1}$ & $\mathbf{5 0 1}$ & $\mathbf{1 . 0 0 1}$ & $\mathbf{2 . 0 0 1}$ & $\mathbf{5 . 0 0 1}$ & $\mathbf{1 0 . 0 0 1}$ & Zara- & Total \\
\hline Escuela de Educación Infantil & $\mathbf{1 0 0}$ & $\mathbf{2 5 0}$ & $\mathbf{5 0 0}$ & $\mathbf{1 . 0 0 0}$ & $\mathbf{2 . 0 0 0}$ & $\mathbf{5 . 0 0 0}$ & $\mathbf{1 0 . 0 0 0}$ & $\mathbf{5 0 . 0 0 0}$ & goza & \\
Colegio de Educación Infantil y Primaria & 0 & 1 & 0 & 1 & $\mathbf{1}$ & 0 & 0 & 1 & 0 & 4 \\
Centro Rural Agrupado (sede) & 4 & $\mathbf{7}$ & $\mathbf{7}$ & 16 & $\mathbf{2 8}$ & $\mathbf{3 3}$ & $\mathbf{1 4}$ & 31 & 56 & $\mathbf{1 9 6}$ \\
Centro Rural Agrupado (unidad) & 0 & 10 & 15 & 31 & $\mathbf{2 1}$ & $\mathbf{1}$ & 0 & 0 & 0 & 78 \\
Instituto de Educación Secundaria & $\mathbf{2 0}$ & 142 & 134 & 80 & $\mathbf{2 9}$ & 2 & 0 & 0 & 0 & 407 \\
Sección de Instituto de Educación Secundaria & 0 & 0 & 0 & 0 & 6 & 19 & 10 & 22 & 29 & 86 \\
Instituto de Formación Profesional & 0 & 1 & 2 & 6 & 2 & 0 & 0 & 0 & 11 \\
\hline
\end{tabular}

Fuente: Gobierno de Aragón. Departamento de Educación y Ciencia.

A diferencia de los anteriores, los CRA constan de una sede central localizada en un determinado punto y un cierto número de "unidades" ubicadas en núcleos de población distintos. Su denominación hace referencia a su origen, las llamadas "escuelas unitarias", que responden a la no menos clásica imagen de escuela rural: una unidad (un aula, un maestro) donde estudia un cierto número de niños de diferentes edades y cursos. Los CRA surgen de la agrupación de estas escuelas unitarias, en un intento de racionalizar al máximo los recursos educativos y la distribución del profesorado. Los $78 \mathrm{CRA}$ que hay en Aragón constan de un total de 407 unidades, lo que da una media de 5 por centro.

Los CRA están localizados en asentamientos rurales, con una sola excepción. Las sedes se ubican preferentemente en los núcleos de mayor tamaño: el 40 por ciento en los de quinientos a mil habitantes, subiendo la proporción hasta el 67 por ciento si se añaden las ubicadas en los núcleos de mil a dos mil. Las unidades siguen una pauta de localización distinta, pues el 67 por ciento del total se distribuye entre los asentamientos de cien a quinientos habitantes.

Al comparar las tablas 1 y 2 se comprueba que a partir de 500 habitantes es mayor el número de centros y unidades de educación infantil y primaria que el de núcleos de población, igualándose ambas cifras en el grupo de 251 a 500, lo cual significa que en los asentamientos cuya población supera los 250 habitantes hay algún centro educativo, aunque sea una unidad de CRA. Por debajo de ese umbral de población, sin embargo, abundan los asentamientos que no cuentan con ninguno: el 43 por ciento de los que tienen entre 101 y 250 habitantes y el 96 por ciento de los de menos de 100.

Por lo que respecta a la educación secundaria, como se recoge en la tabla 2 , hay en Aragón 91 centros públicos: 5 de ellos son Institutos de FP, en los que sólo se cursa el nivel educativo que les da nombre; los 86 restantes son Institutos de Educación Secundaria, es decir, centros en los que se imparten como mínimo los cuatro cursos de la ESO y además, aunque no en todos los casos, el bachillerato y algu- 
nos ciclos formativos de FP. A ello hay que añadir 11 secciones de instituto, ubicadas en localidades distintas de los centros de que forman parte.

La tabla 2 muestra la mayor selectividad espacial de los Institutos de Educación Secundaria con respecto a los centros de los niveles educativos anteriores, pues sólo el 7 por ciento de los mismos aparece localizado en núcleos rurales y, dentro de ellos, precisamente en los de mayor tamaño, los de mil a dos mil habitantes. El 33 por ciento se localiza en núcleos de población intermedios y el 60 por ciento restante en las ciudades. El mapa de la figura 4 muestra la localización de estos centros, así como la de los de primaria que están adscritos a ellos, pudiendo comprobarse fácilmente la menor densidad de la red de centros de secundaria y su ubicación en asentamientos de mayor centralidad y nivel jerárquico.

Las 11 secciones de instituto contribuyen a densificar un poco más la red, acercando estos servicios educativos a mayores extensiones del territorio regional y a núcleos de población de menor rango jerárquico: nueve secciones están localizadas en asentamientos rurales con más de 250 habitantes y las otras dos en núcleos intermedios con menos de cinco mil. En algunas comarcas cuya red de asentamientos es muy laxa son los únicos centros educativos de nivel secundario, mientras que en otras comarcas muy extensas refuerzan los servicios de este nivel permitiendo acortar sensiblemente los desplazamientos de los escolares ${ }^{6}$.

Al comparar los datos de las tablas 1 y 2 se comprueba que a partir de cinco mil habitantes es mayor el número de centros y secciones de educación secundaria que el de núcleos de población, lo cual significa que en los asentamientos cuya población supera dicho valor hay algún centro educativo de este nivel. Por debajo de ese umbral de población, sin embargo, abundan los asentamientos que no cuentan con ninguno: una minoría entre los que tienen de dos a cinco mil habitantes, pero la inmensa mayoría de los asentamientos rurales.

En el mapa de la figura 4 se representan también las áreas que se encuentran a menos de 30 minutos de un centro de enseñanza secundaria, pudiendo observarse que forman una superficie continua en el centro de la comunidad, mientras que en las zonas montañosas aparecen extensiones nada desdeñables al margen de la misma, tanto en los Pirineos como en el Sistema Ibérico, es decir, en las zonas más problemáticas desde el punto de vista territorial por la debilidad de sus redes de asentamientos.

El mayor desafío para la prestación de este nivel educativo se encuentra en estas áreas, que son las más desfavorecidas. Para llevar este servicio hasta aquí en las condiciones necesarias de calidad y oportunidad espacial, se deberá echar mano de todos los medios disponibles, quizás ideando nuevos conceptos de centro, potenciando el uso de tecnologías de la información y cualquier otro que pueda contribuir a este fin. 


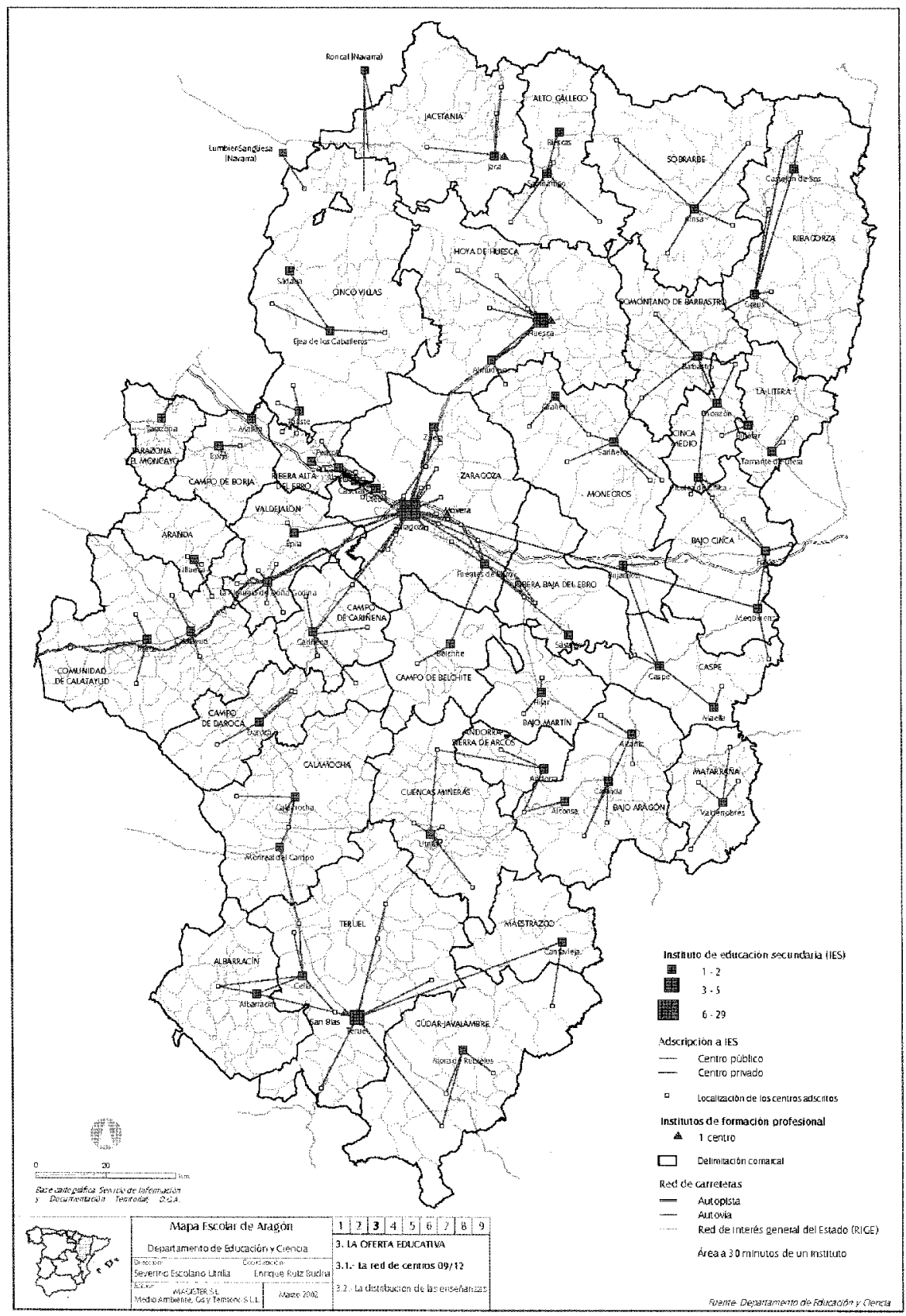

Figura 4. Centros públicos de educación secundaria. Fuente: Escolano et al. (2003, p. 68). 
No obstante, por medio de su red de centros, la administración regional garantiza a toda la población de entre 12 y 16 años la posibilidad de cursar la educación secundaria obligatoria dentro de su propia comarca.

Comparar la situación actual con la de finales de los ochenta resulta problemático por los cambios legislativos que se han producido en este tiempo, fundamentalmente el alargamiento de la escolarización obligatoria de los 14 a los 16 años ${ }^{7}$. El ya citado trabajo de Garcés (1991: 572 y siguientes) contabiliza en Aragón 717 centros públicos de preescolar y educación general básica, mientras que en el 2002 (según la tabla 2) los centros de educación infantil y primaria son 685. Los centros de bachillerato en la primera fecha eran 53, mientras que en la segunda los institutos de educación secundaria eran 86. No ha habido, por tanto, cambios en el número total de centros, porque la disminución de los primeros (32) se compensa con el incremento de los segundos (33).

\section{La red de carreteras y el transporte escolar en Aragón}

Al analizar en el apartado 2 la distribución espacial de la población escolar y en el apartado 3 la de los centros escolares, se ha podido apreciar que los usuarios de los servicios educativos de muchos pequeños asentamientos del medio rural han de desplazarse a diario de su lugar de residencia para acceder a la prestación de unos servicios a los que tienen derecho y que además, en los niveles de educación primaria y ESO, son obligatorios. Para que dichos desplazamientos se produzcan a un coste razonable, medido tanto en tiempo como en dinero, es necesario que los centros escolares sean fácilmente accesibles desde los lugares de residencia de los alumnos, lo cual exige una buena conectividad de las redes de transporte y una organización eficaz del servicio de transporte escolar.

Por lo que respecta a la densidad y calidad de la red de carreteras ${ }^{8}$, como se observa en la figura 5, en Aragón se diferencian claramente tres grandes áreas, reproduciendo el esquema de base topográfica ya observado en la red cie asentamientos: la zona central, que coincide con la Depresión del Ebro, presenta un mayor desarrollo de las infraestructuras de transporte, mientras que las franjas norte y sur, accidentadas por los Pirineos y el Sistema Ibérico, respectivamente, se caracterizan por la escasez de vías de alta capacidad y por bajos índices de conectividad. El área mejor comunicada de Aragón se ordena en franjas concéntricas en torno a una línea imaginaria que uniera Cariñena, Zaragoza y Sariñena, decreciendo la centralidad hacia el norte y hacia el sur (Garrido, 2001). 


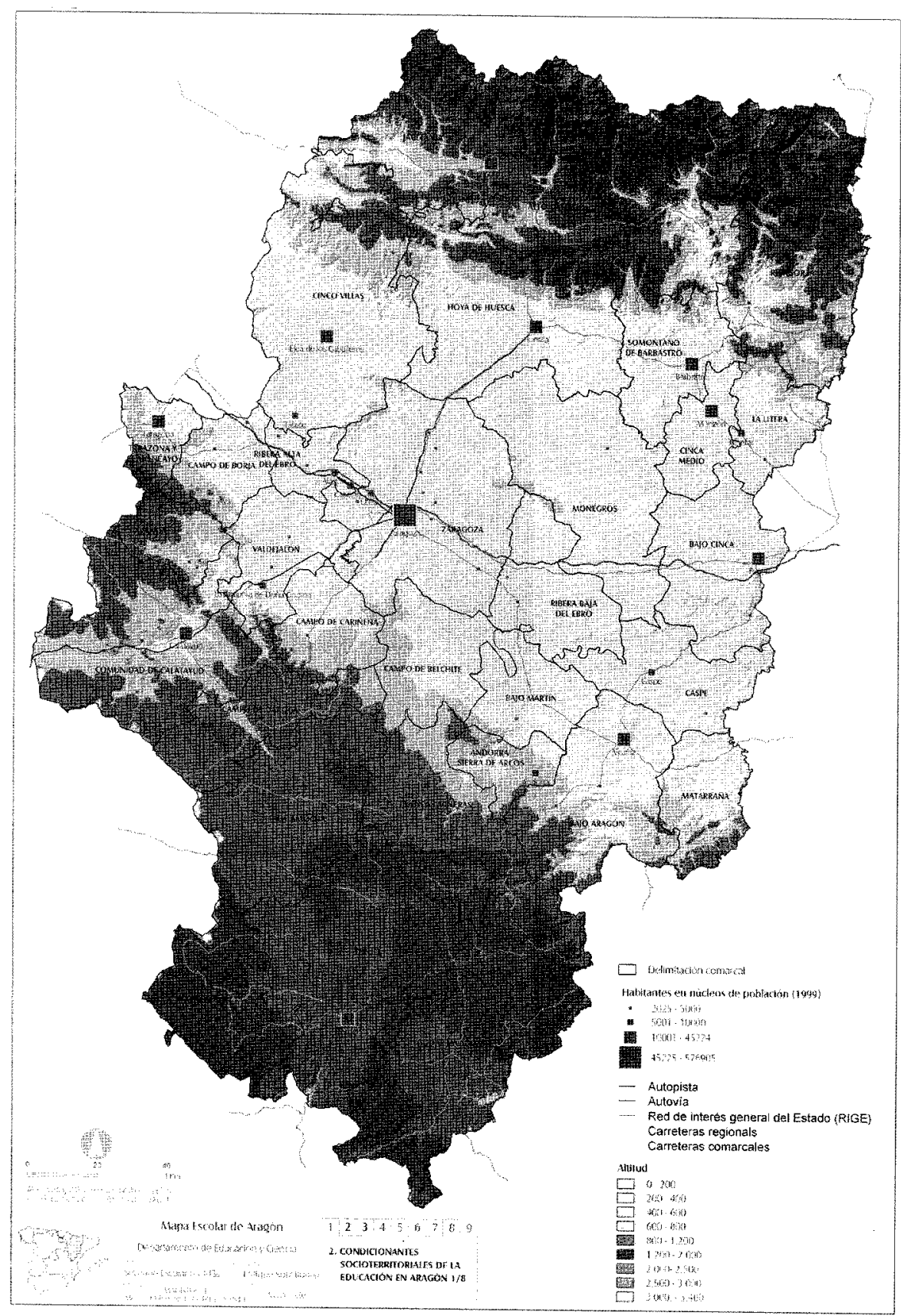

Figura 5. El relieve y la red de transportes.

Fuente: Escolano et al. (2003, p. 47). 
En la zona central, de relieve llano, la elevada densidad de la red de carreteras facilita la formación de abundantes circuitos y la configuración de áreas de servicio con formas compactas. Al contrario, en las áreas montañosas las carreteras, además de presentar más dificultades (puertos y adversidades climáticas), forman redes de baja conectividad, lo que dificulta la formación de áreas de servicio cohesionadas y circulares: éstas adquieren formas elípticas, a veces muy alargadas, cuyo eje mayor se orienta según la dirección de los valles principales. En el caso de los Pirineos la linealidad del sistema de transportes es más acusada, pues las dificultades orográficas y la frontera con Francia hacen que varias carreteras finalicen en "fondos de saco" y no tengan continuidad.

La jerarquía de las carreteras viene marcada por su capacidad portante, que generalmente se mide por la anchura de la plataforma. El nivel más alto corresponde a las vías de gran capacidad (autopistas, autovías y, en general, carreteras de doble calzada), que, como muestra la figura 5, se localizan exclusivamente en el área central de la Depresión del Ebro, y además presentan una marcada estructura radial centrada en la ciudad de Zaragoza.

En el segundo nivel están las carreteras con anchura de plataforma superior a 7 metros, que se identifican en su mayor parte con la red del Estado. Estas vías se concentran fundamentalmente en la Depresión del Ebro, siendo su densidad muy exigua en los Pirineos y en el Sistema Ibérico. En el mapa de la figura 5 puede verse que la proporción del territorio aragonés situada a menos de 2 kilómetros de una carretera nacional es muy reducida (apenas supera el 12 por ciento de la superficie regional).

En definitiva, allí donde la baja densidad de población y su dispersión en asentamientos de pequeño tamaño obliga a seleccionar los núcleos de población en que ubicar los centros escolares, forzando el desplazamiento diario de los alumnos, existen serias limitaciones de la red de carreteras que no favorecen su realización a bajo coste de tiempo y dinero.

En el mapa de la figura 6 se recogen las rutas de transporte escolar de Aragón, con indicación del número de alumnos transportados. En él puede verse que en el área central las rutas son cortas, mientras que en las áreas de montaña del norte y el sur las rutas se alargan considerablemente. Dado que, como indica la figura 5 , en estas últimas las carreteras presentan trazados más sinuosos y menor capacidad portante, la mayor longitud de las rutas de transporte se traduce en un incremento más que proporcional del tiempo empleado en su recorrido, a lo que se suman la inclemencia de los inviernos. 


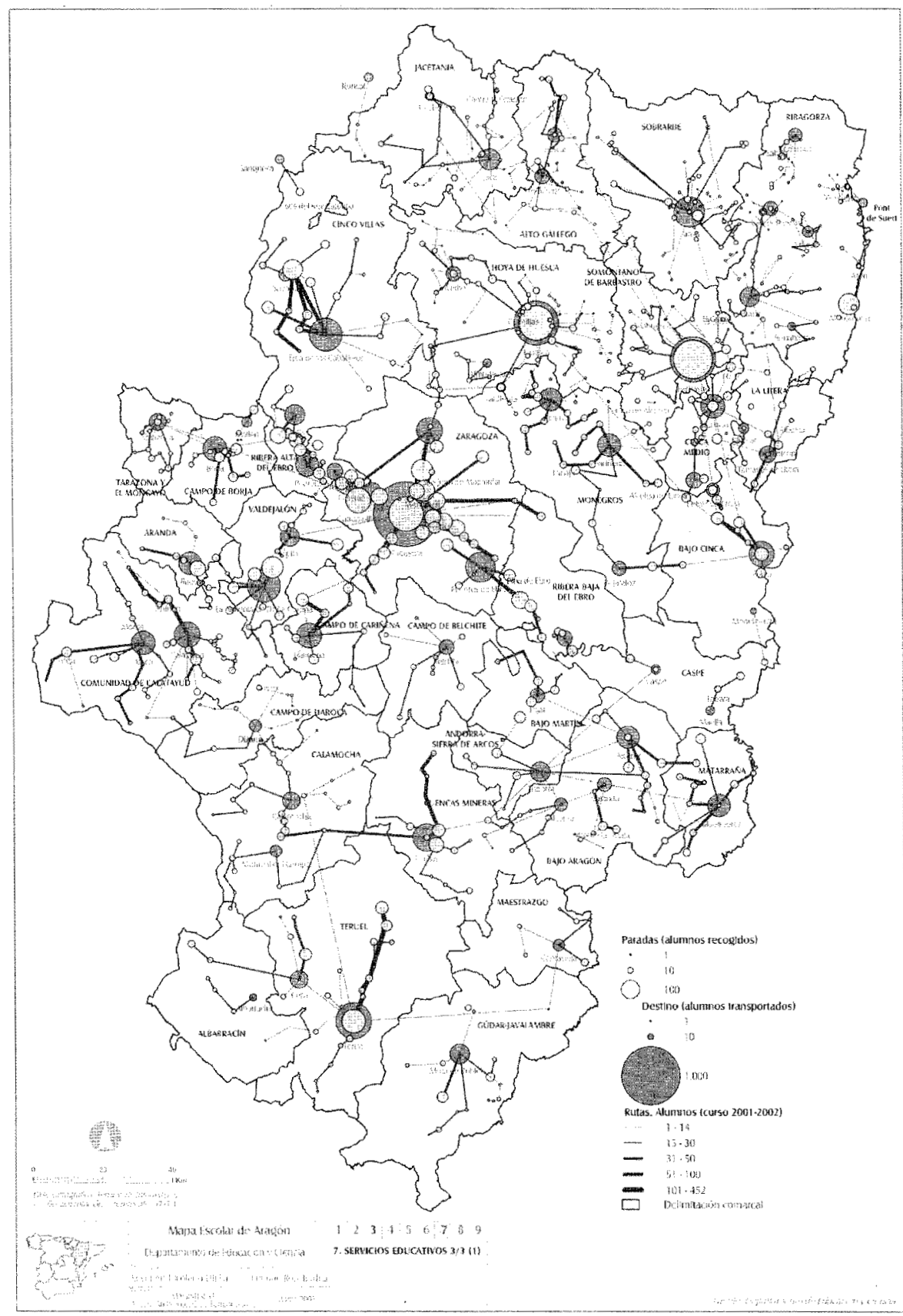

Figura 6. El transporte escolar: alumnos transportados y rutas (2002). Fuente: Escolano et al. (2003, p. 175). 


\section{Algunos elementos de comparación}

Aunque en este trabajo se estudian los servicios educativos, cabe suponer que otros servicios a la población, sobre todo los de carácter dotacional, se encuentran con problemas de organización similares. Aunque cada uno de ellos tiene sus especificidades, puede resultar relevante ensayar alguna comparación para detectar paralelismos y regularidades. Quizá los servicios sanitarios, por su carácter de necesidad básica que el estado debe garantizar, resulten los más adecuados para realizar dicho ensayo comparativo.

Tomando como referencia el trabajo de Escalona y Díez (2003), se observan los siguientes paralelismos entre las redes educativas y sanitarias de Aragón: primero, en las áreas de baja densidad es la iniciativa pública la que garantiza la prestación de dichos servicios, estando casi completamente ausente la iniciativa privada. Segundo: en dichas áreas la distancia de los usuarios a los "centros de salud" (trasunto sanitario de los colegios de primaria) es a menudo tan grande que la administración habilita "consultorios locales" (trasunto de las "unidades" de los CRA en educación) para el desarrollo de las actividades del equipo médico asignado a esa zona en localidades diferentes de las que acogen el centro de salud. Tercero: los centros de salud, que constituyen los equipamientos sanitarios de base, se encuadran geográficamente en "sectores sanitarios", siendo el equipamiento básico de cada uno de éstos un centro hospitalario, al que están adscritos aquéllos, en una dependencia jerárquica similar a la de los centros de educación primaria respecto a los de secundaria; los hospitales se ubican en siete localidades, que corresponden a los niveles más altos de la jerarquía de los asentamientos: la capital regional (Zaragoza), las otras dos capitales de provincia (Huesca y Teruel) y cuatro ciudades pequeñas (Jaca, Barbastro, Calatayud y Alcañiz).

Por otra parte, la comparación de la red de centros educativos de Aragón con la de otras regiones permitiría dar un carácter más general a las conclusiones de este trabajo, pero la tarea no es fácil por dos tipos de razones: unas son de carácter metodológico y derivan de que, como se señalado, los mapas escolares han sido realizados por los gobiernos de las comunidades autónomas, según criterios propios, no siempre coincidentes, en función de la finalidad perseguida en su elaboración. Otras razones que dificultan la comparación derivan de la propia estructura territorial de las regiones. No obstante, con todas las limitaciones, vale la pena ensayar una breve comparación con Cataluña:

Esta región presenta una estructura territorial muy diferente de la aragonesa, pero tiene también algunas áreas con poca densidad de población y una red de carreteras poco trabada. Es el caso, por ejemplo, de la Alta Ribagorza ubicada en los Pirineos: esta comarca tiene un único centro de secundaria, que se encuentra en la capital, 
Pont de Suert, situada al sur, es decir, en la zona más baja del valle; hay cinco centros de primaria, que se hallan en la capital y en otras cuatro localidades, pero en todas las demás no hay ninguno, de manera que un número significativo de escolares debe desplazarse a diario recorriendo distancias nada desdeñables (Mapa escolar de Catalunya); es un caso muy similar al de varias comarcas aragonesas, como se ha visto en los apartados anteriores. El Pallars Sobirá, comarca catalana vecina de la anterior y de estructura territorial parecida, es más extensa y alargada, por lo que las distancias desde la capital, Sort, se incrementan tanto que las autoridades educativas han optado por establecer un segundo centro de secundaria en la localidad de Esterri d'Aneu, al norte del valle, coincidiendo con la anterior en lo que respecta a los centros de primaria; es el mismo caso que la Ribagorza aragonesa donde el instituto de la capital, Graus, muy excéntrica hacia el sur de la comarca, se complementa con la sección de instituto de Castejón de Sos, situada cerca de la cabecera del valle. En esas dos comarcas catalanas, lo mismo que en muchas aragonesas, no hay centros de enseñanza privados, por lo que es la administración pública la que garantiza el derecho a la educación.

Pero las comarcas citadas son más bien excepcionales en Cataluña, cuya densidad de población es muy elevada. Por eso, en la mayor parte de su territorio la red de centros es notablemente más densa que la de Aragón y el problema de la accesibilidad se plantea en términos muy distintos.

\section{Conclusiones}

A lo largo del trabajo ha quedado de manifiesto que la prestación de los servicios educativos en condiciones de equidad y eficiencia se enfrenta en Aragón a una serie de desequilibrios territoriales, que pueden resumirse de la siguiente manera:

- La existencia de extensas áreas con una densidad de población inferior a 5 habitantes por kilómetro cuadrado, muy próxima a lo que podría considerarse el desierto demográfico, cuya superficie se amplía considerablemente si se consideran también las que tienen densidades comprendidas entre 5 y 15 habitantes por kilómetro cuadrado. El problema adquiere caracteres más agudos si se tiene en cuenta el elevado grado de envejecimiento de la población de dichas áreas.

- Una red de asentamientos desequilibrada, en la que son mayoría los núcleos de población de menos de 100 habitantes, con una red urbana fuertemente primacial, cuya ciudad principal alberga a la mitad de la población regional, y una baja densidad de núcleos urbanos e intermedios, irregularmente distribuidos 
por el territorio, de modo que extensas áreas carecen casi por completo de asentamientos de una y otra clase.

- La existencia de amplias porciones de territorio con muy baja densidad de infraestructuras de transporte y carencia completa de vías de gran capacidad.

Por lo que respecta a la prestación de los servicios educativos, la iniciativa privada aplica más bien la lógica del mercado que la del equilibrio territorial, pues la mayor parte de sus centros escolares se localizan en los núcleos de población de mayor tamaño, que es donde se concentra la demanda, especialmente en la ciudad principal.

La iniciativa pública se muestra sensible a los desequilibrios territoriales y mantiene una red de centros accesible a la totalidad de la población en edad escolar, atendiendo a los preceptos legales que garantizan el derecho a la educación y establecen niveles de enseñanza obligatoria. Esto se concreta en lo siguiente:

- Todos los núcleos de población urbanos e intermedios y la gran mayoría de los asentamientos rurales de más de mil habitantes cuentan con al menos un centro de educación infantil y primaria.

- Las unidades de los CRA llevan los servicios educativos de educación infantil y primaria a prácticamente todos los asentamientos de más de 250 habitantes.

- Todos los núcleos de población de más de cinco mil habitantes y las dos terceras partes de los que tienen entre dos mil y cinco mil cuentan con al menos un instituto de educación secundaria.

- Por medio de secciones de instituto se densifica la red de centros de este nivel, permitiendo que todas las comarcas cuenten con servicios de educación secundaria, al menos en su segmento obligatorio (la ESO).

La red de centros escolares, en conclusión, intenta acercar los servicios educativos lo más posible a los usuarios, pero aun con ello sigue habiendo serios desajustes entre la distribución espacial de dicha red y la de la red de asentamientos.

Dichos desajustes se compensan por medio de los servicios de transporte escolar, que en algunas zonas se organizan en rutas de notable longitud, a menudo por carreteras cuyo trazado y anchura obliga a circular a velocidad lenta. Esto alarga notablemente los tiempos de desplazamiento, que alcanzan valores difícilmente tolerables en algunas zonas del territorio aragonés que se encuentran a más de media hora de viaje de un centro de educación secundaria.

La cartografía presentada permite visualizar con toda claridad las áreas más críticas desde el punto de vista de los desequilibrios territoriales señalados: se trata de las franjas septentrional y meridional de la región, que se encuentran accidentadas por relieves montañosos. Es en ellas donde se encuentran las redes de centros más laxas y las rutas de transporte más largas. 


\section{Agradecimientos}

Este artículo tiene su origen en el proyecto PO26/99-HS, "Planificación y gestión de la enseñanza no universitaria en Aragón mediante un sistema de información geográfica", financiado por el Departamento de Educación y Ciencia del Gobierno de Aragón.

\section{Notas}

1 La población escolar comprende a todos los alumnos que cursan las enseñanzas escolares de régimen general. No obstante, en la tabla 1 se incluye sólo la población escolar de los niveles obligatorios, que es un subconjunto de la anterior, por ser la que plantea los problemas más graves de organización de la red de centros educativos, precisamente por la obligatoriedad de dichos niveles.

2 Mientras que en la tabla 1 se recogen los alumnos de educación obligatoria según la legislación actual (6 a 16 años), en el trabajo citado se recoge la población del alumnado de Educación General Básica, que era el único nivel entonces obligatorio ( 6 a 14 años). Aunque las edades no coinciden, los clatos son perfectamente comparables puesto que se refieren en ambos casos a los años en que la escolarización es obligatoria.

3 En Escolano (1999: 65) se utilizan diversas variables para medir el envejecimiento demográfico y en todas ellas los valores de Aragón respecto a los de España son claramente indicativos de que la población regional está más envejecida que la nacional. A título de ejemplo, el índice de envejecimiento de VeyretVerner alcanza el valor 1 en Aragón, frente a 0,7 en España, considerándose envejecida a todá población cuyo indice supere el valor 0,4 .

4 En Aragón existen 82 centros privados en los que se cursan de forma integrada niveles de educación infantil, primaria y secundaria. Para homogeneizar los datos con los relativos a los de titularidad pública, pues en este caso los centros de secundaria son independientes de los de infantil y primaria, aunque en ocasiones comparten edificios e instalaciones, los 82 centros privados se han contabilizado dos veces, una en el grupo de infantil y primaria y otra en el de secundaria.

5 Se trata de las comarcas de Ribagorza, Campo de Belchite y Matarraña, más las altas Cinco Villas, en el Prepirineo occidental.

6 Las comarcas cuya red de asentamientos es muy laxa son: Campo de Belchite, Albarracín, Maestrazgo y Ribera Baja del Ebro. En Alto Gállego, Ribagorza y Cinco Villas las secciones de Biescas, Castejón de Sos y Sádaba, respectivamente, sirven a los escolares de las tierras altas de cada comarca.

7 A finales de los 80 la escolarización obligatoria incluía un único nivel, la educación general básica (de 6 a 14 años), mientras que ahora incluye dos niveles distintos, la educación primaria (de 6 a 12 años) y la educación secundaria obligatoria (de 12 a 16 años).

8 El transporte por ferrocarril podría complementar a las carreteras e incluso tener un papel dominante en la articulación de algunas áreas educativas. Pero la red ferroviaria es muy poco densa y, salvo por lo que respecta a la línea Madrid-Zaragoza-Barcelona, las infraestructuras no se encuentran en buen estado. 


\section{Bibliografía}

Beavon, K.S.O. (1981): Geografia de las actividades terciarias. Una reinterpretación de la teoría de los lugares centrales. Barcelona, Oikos-tau.

Berry, B.J.L. (1972): Geografia de los centros de mercado y distribución al por menor. Barcelona, Vicens Vives (edición original inglesa: 1967).

Christaller, W. (1966): Central places in southern Germany. Englewood Cliffs, Prentice Hall (edición original alemana: 1933).

Escalona Orcao, A.I. y Díez Cornago, C. (2003): Accesibilidad geográfica de la población rural a los servicios básicos de salud: estudio en la provincia de Teruel. Ager, 3, p. 111-149.

Escolano, S. (1999): La población aragonesa en el umbral del siglo XXI. Zaragoza, Caja de Ahorros de la Inmaculada.

Escolano Utrilla, S. et al. (2003): Mapa Escolar de Aragón. Zaragoza, Gobierno de Aragón.

Garcés Campos, R. (dir. y coord.) (1991): Mapa escolar de Aragón. Zaragoza, Diputación General de Aragón.

Garrido, J. (2001): El transporte como factor de desarrollo en Aragón. Zaragoza, Institución Fernando el Católico.

Hallak, J. (1978): El mapa escolar, un instrumento de la politica de educación. Paris, UNESCO.
Jean, Y. (1995): École et aménagement du territoire rural: quel avenir pour les petites structures scolaires et les communes rurales? Annales de Géograpbie, 583, p. 236-255.

L'efecte de la reforma educativa sobre la xarxa escolar a la provincia de Barcelona. Barcelona, Diputació de Barcelona, 1990.

Mapa escolar de Catalunya. Situació actual $i$ descripció per comarques. Barcelona, Generalitat de Catalunya, 1989.

Moreno Jiménez, A. (1995): Planificación y gestión de servicios a la población desde la perspectiva territorial: algunas propuestas metodológicas. Boletín de la Asociación de Geógrafos Españoles, 20, p. 115-134.

Pitarch, M.D. (2000): Los modelos de planificación espacial de los servicios públicos: el caso de los servicios educativos. Cuadernos de Geografía, 67-68, p. 119-136.

Requés, P. (dir.) (2000): Mapa escolar de Cantabria. Estudio de base para la planificación de los servicios educativos no universitarios de la región. Santander, Gobierno de Cantabria.

Salom Carrasco, J. y Albertos Puebla, J.M. (2000): El impacto territorial de la planificación educativa: el mapa escolar de aplicación de la LOGSE en la Comunidad Valenciana. Cuadernos de Geografia, 67-68, p. 37-56. 